University of Nebraska - Lincoln

DigitalCommons@University of Nebraska - Lincoln

April 1991

\title{
Biological Effectiveness of High-Energy Protons: Target Fragmentation
}

\author{
Francis A. Cucinotta \\ Space Transportation Systems Division, Rockwell International, Houston, Texas, \\ francis.cucinotta@unlv.edu \\ Robert Katz \\ University of Nebraska-Lincoln, rkatz2@unl.edu \\ John W. Wilson \\ NASA Langley Research Center, Hampton, Virginia \\ Lawrence W. Townsend \\ NASA Langley Research Center, Hampton, Virginia \\ Judy Shinn \\ NASA Langley Research Center, Hampton, Virginia \\ See next page for additional authors
}

Follow this and additional works at: https://digitalcommons.unl.edu/physicskatz

Part of the Physics Commons

Cucinotta, Francis A.; Katz, Robert; Wilson, John W.; Townsend, Lawrence W.; Shinn, Judy; and Hajnal, Ferenc, "Biological Effectiveness of High-Energy Protons: Target Fragmentation" (1991). Robert Katz Publications. 10.

https://digitalcommons.unl.edu/physicskatz/10

This Article is brought to you for free and open access by the Research Papers in Physics and Astronomy at DigitalCommons@University of Nebraska - Lincoln. It has been accepted for inclusion in Robert Katz Publications by an authorized administrator of DigitalCommons@University of Nebraska - Lincoln. 


\section{Authors}

Francis A. Cucinotta, Robert Katz, John W. Wilson, Lawrence W. Townsend, Judy Shinn, and Ferenc Hajnal 


\title{
Biological Effectiveness of High-Energy Protons: Target Fragmentation
}

\author{
Francis A. Cucinotta, ${ }^{*, 1}$ Robert Katz, $†$ John W. Wilson, $\ddagger$ LaWrence W. TownSend $\ddagger$ \\ JUDY SHINN, $\ddagger$ AND FERENC HAJNAL $§$
}

*Space Transportation Systems Division, Rockwell International, Houston, Texas 77058; †University of Nebraska, Lincoln, Nebraska 68588-0111; $\ddagger N A S A$ Langley Research Center, Hampton, Virginia 23665-5225; and §Environmental Measurements Laboratory,

U.S. Department of Energy, New York, New York 10014

Cucinotta, F. A., Katz, R., Wilson, J. W., Townsend, L. W., ShinN, J., AND HAJNAL, F. Biological Effectiveness of High-Energy Protons: Target Fragmentation. Radiat. Res. 127, 130-137 (1991).

High-energy protons traversing tissue produce local sources of high-linear-energy-transfer (LET) ions through nuclear fragmentation. We examine the contribution of these target fragments to the biological effectiveness of high-energy protons using the cellular track model. The effects of secondary ions are treated in terms of the production collision density using energy-dependent parameters from a high-energy fragmentation model. Calculations for mammalian cell cultures show that at high dose, at which intertrack effects become important, protons deliver damage similar to that produced by $\gamma$ rays, and with fragmentation the relative biological effectiveness (RBE) of protons increases moderately from unity. At low dose, where sublethal damage is unimportant, the contribution from target fragments dominates, causing the proton effectiveness to be very different from that of $\gamma$ rays with a strongly fluence-dependent RBE. At high energies, the nuclear fragmentation cross sections become independent of energy. This leads to a plateau in the proton single-particle-action cross section, below $1 \mathrm{keV} / \mu \mathrm{m}$, since the target fragments dominate. (๑) 1991 Academic Press, Inc.

\section{INTRODUCTION}

Human exposure to ionizing radiation is most often at low doses at which more than one particle is not likely to traverse individual cells. The general population is exposed to very low levels of the highly ionizing particles of galactic cosmic rays (GCR) (1). Astronauts are also exposed to very low fluences of GCR (2), but the major exposure comes from trapped particle radiation. Solar flares can contribute a small increment to the total exposure, the dose depending on the orbital inclination. The high-linear-energy-transfer (LET) component of the cosmic rays is of principal concern because of the lack of human data (3) for assessing their effects. Ongoing efforts are being made to study the effects

\footnotetext{
${ }^{1}$ Present address: NASA Langley Research Center, Hampton, VA 23665.
}

of these high-LET components (4-6), although much work remains, especially at low dose levels. However, the major component of the cosmic rays is low-LET protons, representing about $90 \%$ of the cosmic-ray flux. Nuclear fragmentation effects are also seen to be important in assessing exposures to heavy ions, with protons produced with the greatest frequency in such events. An understanding of the biological effects of high-energy protons is then required at the level of single-particle action for a comprehensive assessment of the risks from GCR.

The relative biological effectiveness (RBE) of high-energy protons has been observed to be similar to that of $\mathrm{X}$ rays or ${ }^{60} \mathrm{Co} \gamma$ rays in a variety of experiments with mammalian cell cultures and animals (see, for example, Refs. (7-10)), with some variation in effectiveness observed for different biological end points, dose levels, dose rates, and postirradiation time (see Ref. (7) for RBE values for protons). This similarity between proton effectiveness and that of $X$ or $\gamma$ irradiation is attributed to the resemblance between the spatial and energy distribution of secondary electrons from low-LET protons and the distribution of electrons from gamma rays. It has been noted that the contributions of high-LET secondary ions increase the proton RBE from unity in comparison to $\gamma$ rays in clinical therapy studies $(7$, 8 ). In survival experiments with Chinese hamster cells irradiated with $160-\mathrm{MeV}$ protons at the plateau region of the Bragg curve and in a spread-out Bragg peak (10), a rising RBE with decreasing dose was seen below doses of $2 \mathrm{~Gy}$. A limiting maximum in the RBE was predicted (10) for smaller values of proton dose, based on the assumption of a linear-quadratic dose-response model. The RBE increases as the dose of the reference radiation is decreased until the responses to both the reference low-LET radiation and the high-LET radiation become linear $(6,11)$. This would also hold for the RBE of nuclear fragments with proton irradiation. We note that in experiments to date, survival data for proton irradiations seldom extend to doses below $1 \mathrm{~Gy}$.

Energetic protons passing through tissue produce local sources of high-LET fragmentation products which should contribute to the proton effect. Since production of $\delta$ rays causes biological damage, the modification to the local dis- 
tribution of $\delta$ rays which scales as $Z^{* 2} / \beta^{2}$, where $Z^{*}$ is the effective charge number and $\beta$ the velocity, may be significant for the low-energy, higher-charge secondaries in tissues. We expect the fragmentation contribution to be least important at high dose or fluence, where many protons will pass through a single cell. Here, the direct ionization from primary protons is adequate for producing damage without the help of the local secondaries. It is in the initial portion of biological response curves where we expect the secondary ions to be most important. With the action of single protons representing a small probability for producing the biological effect, the high-LET fragments will dominate if nuclear production cross sections are sufficiently large.

The cellular track model of Katz et al. (12-14) describes both the intratrack and intertrack action of ions on biological systems through the ion-kill and $\gamma$-kill modes of cellular damage. The model is parametric, relying on survival curves for $\mathrm{X}$ rays or $\gamma$ rays and track-segment irradiations with heavy ions for its parameters with the underlying formalism allowing for generalizations to arbitrary ions. The track model has enjoyed a variety of successes in describing and predicting effects in a host of biological systems. In working with a parametric model, a mechanistic description of the cellular response to radiation is avoided, and cell-cycle dependence and repair of sublethal damage are not considered. In this paper, we introduce modifications to the track model to account for the effects of target fragmentation in determining the biological effectiveness of high-energy protons. Fragmentation contributions are described, using a recently developed nuclear fragmentation model (15). The fragmentation parameters are expected to contain some uncertainties, because measurements are scarce. The dependence of the proton RBE on energy is studied using cellular response parameters for Chinese hamster cells (14). An effective action cross section for the combined effects of protons and tissue secondaries (nuclear stars) is considered. Comparisons are made to the survival measurements of Ref. (10) in the plateau region of the Bragg curve using the Katz model and the high-energy baryon transport code, BRYNTRN (16).

\section{CELLULAR TRACK MODEL}

The cellular track model of Katz has been described extensively (12-14). Here, we outline its basic concepts and discuss the inclusion of target fragmentation for proton irradiations. The track model attributes biological damage from energetic ions to the secondary electrons ( $\delta$ rays) produced along the ion path. Cell inactivation is separated into a so-called grain-count regime, where inactivation occurs randomly along the path of the particle, and into the socalled track-width regime, where many inactivations occur and are said to be distributed like a "hairy-rope." Four cellular parameters describe the response of the cells, two of which ( $m$, the number of targets per cell, and $D_{0}$, the char- acteristic X-ray dose) are extracted from the response of the cellular system to $\mathrm{X}$ and $\gamma$ irradiation. The other two $\left(\sigma_{0}\right.$ interpreted as the cross-sectional area of the cell nucleus within which the inactivation sites are located, and $\kappa$, a measurement of the size of the inactivation site) are found principally from survival measurements after track-segment irradiations with energetic charged particles. The transition from the grain-count regime to the track-width regime takes place at a $Z^{* 2} / \kappa \beta^{2}$ of about 4 : at lower values we are in the grain-count regime, and at higher values the trackwidth regime.

To accommodate for the capacity of cells to accumulate sublethal damage, two modes of inactivation are identified: "ion-kill" (corresponding to intratrack effects) and "gamma-kill" (corresponding to intertrack effects). When the passage of a single ion damages cells, the ion-kill mode occurs. In the grain-count regime the fraction of cells damaged in the ion-kill mode is taken as $P=\sigma / \sigma_{0}$, where $\sigma$ is the single-particle inactivation cross section, and $P$ is the probability of damage in the ion-kill mode. The track model assumes that a fraction of the ion dose, $(1-P)$, acts cumulatively with that from other particles to inactivate cells, in the gamma-kill mode. The surviving fraction of a cellular population $N_{0}$, whose response parameters are m, $D_{0}, \sigma_{0}$, and $\kappa$ after irradiation by a fluence of particles $F$, is written (12)

$$
\frac{N}{N_{0}}=\pi_{i} \times \pi_{\gamma},
$$

where

$$
\pi_{i}=e^{-\sigma F}
$$

is the ion-kill survival probability and

$$
\pi_{\gamma}=1-\left(1-e^{-D_{\gamma} / D_{0}}\right)^{m}
$$

is the gamma-kill survival probability. The gamma-kill dose fraction is

$$
D_{\gamma}=(1-P) D,
$$

where $D$ is the absorbed dose. The single-particle inactivation cross section $\sigma$ is taken in the grain count regime as

$$
\sigma=\sigma_{0}\left(1-e^{-Z^{* 2} / \alpha \beta^{2}}\right)^{m}
$$

where the effective charge number is given by

$$
Z^{*}=Z\left(1-e^{-125 \beta / Z^{2 / 3}}\right)
$$

In the track-width regime, we use the approximation from Roth and Chang ${ }^{2}$

\footnotetext{
${ }^{2}$ R. A. Roth and Y. L. Chang, private communication, 1977.
} 


$$
\sigma=\sigma_{0}\left\{1+\left(C_{1}+C_{2} m+C_{3} m^{2}\right)\left(\frac{Z^{* 2}}{\kappa \beta^{2}}\right)^{1.4}\right\},
$$

where $C_{1}=0.0393517, \quad C_{2}=-0.130822, \quad$ and $C_{3}$ $=0.00137756$, which allows for $\sigma>\sigma_{0}$, but does not represent the "hook" structure observed (14) for very high-LET ions. In the track-width regime, $P>1$, we take $\pi_{\gamma}=1$.

The RBE at a specific survival level is given by

$$
\mathrm{RBE}=D_{x} / D,
$$

where

$$
D_{x}=-D_{0} \ln \left[1-\left(1-N / N_{0}\right)^{1 / m}\right]
$$

is the X-ray dose at which this level is obtained. Of particular interest is the low fluence limit of Eq. (8). For low doses, $D \ll D_{0}$, where only single-track effects occur, the RBE is approximately (17)

$$
\mathrm{RBE}=D_{0}\left(\frac{\sigma}{\mathrm{LET}}\right)^{1 / m} D^{(1 / m-1)} .
$$

We next extend the track model to include the effects of target fragmentation in tissue.

\section{TARGET FRAGMENTATION}

High-energy protons passing through tissue will occasionally suffer nuclear reactions that produce low-energy, high-LET ions from the tissue itself. The target fragments, in turn, will be a source of $\delta$-ray production which may contribute to biological damage locally in the tissue matrix. Using collision density formalism, the differential fluence $(15,18)$ describing the local source of target fragments can be rewritten as

$$
F_{j}=\frac{1}{S_{j}(E)} \int_{E}^{\infty} \frac{d \Sigma_{j}\left(E^{\prime}\right)}{d E^{\prime}} F_{\mathrm{p}}\left(E_{\mathrm{p}}\right) d E^{\prime},
$$

where $j$ is the fragment label, $S$ the stopping power or LET, $\Sigma_{j}$ the macroscopic nuclear production cross section, and $F_{\mathrm{p}}$ the fluence of protons with energy $E_{\mathrm{p}}$. An effective action cross section for the proton including the target fragments (nuclear stars) is now written as

$$
\sigma^{*}=\sigma_{\mathrm{p}}\left(E_{\mathrm{p}}\right)+\frac{1}{F_{\mathrm{p}}\left(E_{\mathrm{p}}\right)} \sum_{j} \int_{0}^{\infty} F_{j}\left(E_{j}\right) \sigma_{j}\left(E_{j}\right) d E_{j},
$$

where $\sigma_{\mathrm{p}}$ and $\sigma_{j}$ are given by the Katz formalism (Eqs. (5)(7)). The gamma-kill dose for the proton-plus-target fragments is written

$$
D_{\gamma}=D_{\gamma \mathrm{p}}\left(E_{\mathrm{p}}\right)+\sum_{j} \int_{0}^{\infty} F_{j}\left(E_{j}\right) S_{j}\left(E_{j}\right)\left(1-P_{j}\left(E_{j}\right)\right) d E_{j} .
$$

The production energy spectra for the target fragments are expressed as (15)

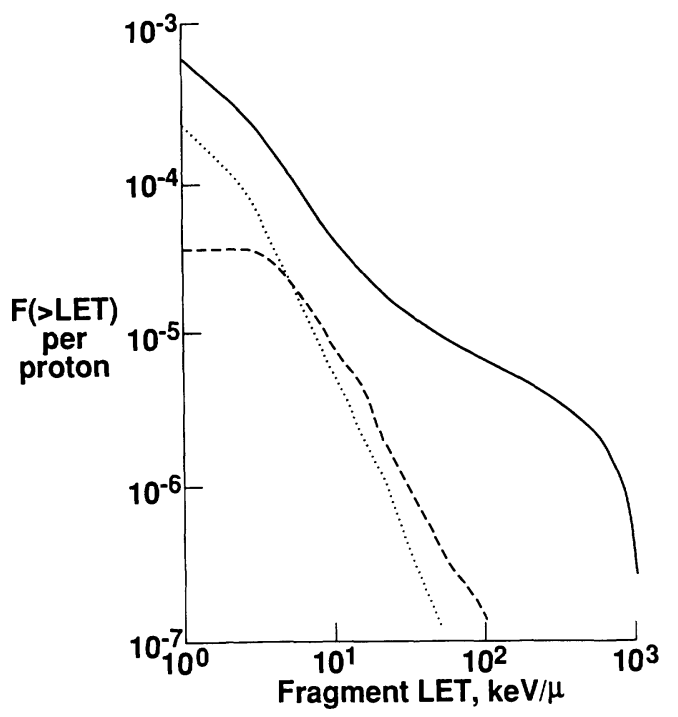

FIG. 1. Integral LET spectra [ $F(>$ LET) per proton] for nuclear fragments produced by $1-\mathrm{GeV}$ protons in water as a function of fragment LET $(\mathrm{keV} / \mu \mathrm{m})$. Solid line, all fragments; dotted line, secondary protons; and dashed line, secondary $\alpha$ particles.

$$
\frac{d \Sigma_{j}}{d E}=\frac{\Sigma_{j}(\sqrt{E})}{\left(2 \pi E_{0 j}^{3}\right)^{1 / 2}} e^{-E / 2 E_{0 j}}
$$

where $3 E_{0 j}$ is the average energy of the fragment.

The fragmentation parameters used are discussed in Ref. (15). The cross sections for light-ion production are from the results of Bertini using the Monte Carlo code (19) and the Silberberg-Tsao empirical model is used for the heavier fragments (20). The average energy of the tissue fragments is related to the momentum width measured experimentally (21), which Wilson et al. (15) fitted empirically. We note that the largest uncertainties exist for light-ion production $(A=2$ and 9$)$ and for energies below $100 \mathrm{MeV}$. Elastic recoils and meson production, above several hundred $\mathrm{MeV}$, will also contribute and should be added. The stopping power in tissue is from the work of Wilson et al. (22) based on the analysis of Ziegler (23).

The solid line in Fig. 1 displays the fragment LET component from 1-GeV protons in tissue derived from Eq. (11). The dotted and dashed lines show the contributions from proton-fragment and $\alpha$ secondaries, respectively.

The proton action cross section for cell survival of Chinese hamster cells is shown in Fig. 2 as a function of the proton energy. The cellular response parameters (14) are $D_{0}$ $=1.82 \mathrm{~Gy}, m=3, \sigma_{0}=4.28 \times 10^{-7} \mathrm{~cm}^{2}$, and $\kappa=1100 \mathrm{as}$ fitted to the data from Skarsgard et al. (24). Discussed below are comparisons to the data of Hall et al. (10) for survival of Chinese hamster cells where the characteristic Xray dose, $D_{0}$, is taken as $2.9 \mathrm{~Gy}$, as found from their X-ray data, and with the remaining parameters the same as given by Katz et al. (14). The dotted line in Fig. 2 shows the 


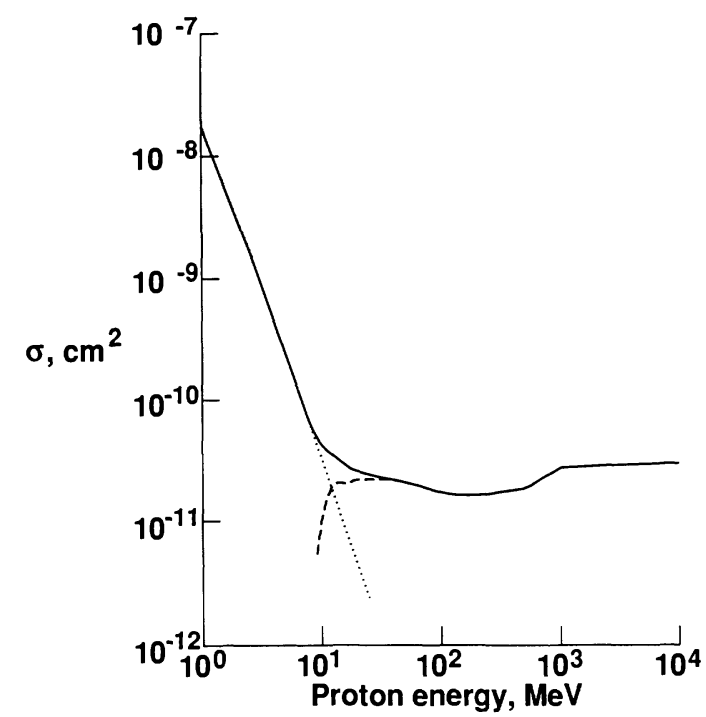

FIG. 2. Calculated values of the proton action cross section for survival of Chinese hamster cells as a function of the proton energy. Dotted line is primary proton contribution, dashed line is the tissue fragmentation contribution, and solid line is the total.

contributions from primary ionization; the dashed line shows contributions from secondary ions. We note that the oxygen and nitrogen fragments contribute partially to the cross section in the track-width regime. The decreasing proton LET with increasing energy is seen to lead to complete domination by target fragments above about $50 \mathrm{MeV}$. The shape of the action cross section in Fig. 2 directly reflects the nuclear absorption cross section in tissue. We expect a further increase above several hundred $\mathrm{MeV}$ when meson production is included in the cross section. In Table $\mathrm{I}$, the individual contributions to the action cross section are shown for several proton energies. Secondary protons and $\alpha$ 's are dominant with a broad spectrum of fragments making minimal contributions. The primary proton makes up an insignificant fraction of the action cross section above $100 \mathrm{MeV}$, and the relatively slow change with energy of the nuclear production cross sections leads to a plateau in the action cross section at high proton energies.

Figure 3 shows the action cross section as a function of proton LET with the calculations of Fig. 2, extended down to $0.1 \mathrm{MeV}$, corresponding to high-LET protons. The behavior of the cross section below $0.5 \mathrm{keV} / \mu \mathrm{m}$ shows the dominance of the tissue secondaries (nuclear stars). At about $0.2 \mathrm{keV} / \mu \mathrm{m}$, the proton LET reaches a minimum and then increases, which is the origin of the hook in Fig. 3 at the lowest LET values. It would be interesting to test our results for the proton cross section by experiment. Results herein assume an equilibrium in the local secondary fluence spectra, and are sensitive to interface effects (25) and the composition of the host media of the cell culture.

In Fig. 4 we show the proton gamma-kill dose divided by $D_{0}$ as a function of proton energy. The results for the pri- mary ionization are shown by the dotted line and the effects of fragments are included in the solid line. The production of secondary ions is seen to have a negligible effect on intertrack effects, except at the highest energies where a small contribution is seen.

\section{SURVIVAL CURVES AND THE PROTON RBE}

Cell survival curves for $10-, 100-$, and $1000-\mathrm{MeV}$ protons are shown as a function of absorbed dose in Figs. 5-7. The solid lines include the target fragment terms and the dotted lines neglect their contributions. Results show the importance of the production of secondary ions for increasing energy. We note, for example, that at $1000 \mathrm{MeV}$ the increase in cell death due to the fragments does not lead to substantial changes in RBE at high doses as can be calculated from Fig. 7. The RBE based on the initial portion of the survival curves for proton and $\gamma$ irradiation may be large. The RBE versus dose is shown in Fig. 8 with all curves including the effects of target fragmentation. We note that

TABLE I

Target Fragment Contributions to Proton Action Cross Section for V79 Chinese Hamster Cells $\sigma, 10^{-12} \mathrm{~cm}^{2}$

\begin{tabular}{|c|c|c|c|c|}
\hline \multirow[b]{2}{*}{$Z f$} & \multirow[b]{2}{*}{$A f$} & \multicolumn{3}{|c|}{$E_{\mathrm{p}}(\mathrm{MeV})$} \\
\hline & & 10 & 100 & 1000 \\
\hline 1 & 1 & 4.58 & 6.70 & 7.14 \\
\hline 1 & 2 & 0.21 & 0.40 & 1.49 \\
\hline 1 & 3 & 0.10 & 0.19 & 0.22 \\
\hline 2 & 3 & 0.16 & 0.34 & 0.66 \\
\hline 2 & 4 & 1.68 & 3.59 & 12.22 \\
\hline 3 & 5 & 0.32 & 0.42 & 0.65 \\
\hline 3 & 6 & 0.46 & 0.53 & 0.68 \\
\hline 3 & 7 & 0.04 & 0.17 & 0.42 \\
\hline 4 & 6 & 0.01 & 0.07 & 0.20 \\
\hline 4 & 7 & 0.36 & 0.42 & 0.49 \\
\hline 4 & 8 & 0.41 & 0.51 & 0.53 \\
\hline 4 & 9 & $<0.01$ & 0.04 & 0.09 \\
\hline 5 & 8 & $<0.01$ & 0.04 & 0.11 \\
\hline 5 & 9 & 0.10 & 0.30 & 0.32 \\
\hline 5 & 10 & 0.22 & 0.35 & 0.28 \\
\hline 5 & 11 & 0.02 & 0.21 & 0.35 \\
\hline 6 & 10 & $<0.01$ & 0.03 & 0.08 \\
\hline 6 & 11 & 0.04 & 0.41 & 0.32 \\
\hline 6 & 12 & 0.50 & 1.00 & 0.63 \\
\hline 6 & 13 & 0.14 & 0.32 & 0.24 \\
\hline 6 & 14 & $<0.01$ & 0.02 & 0.03 \\
\hline 7 & 12 & $<0.01$ & 0.01 & 0.04 \\
\hline 7 & 13 & 0.03 & 0.09 & 0.07 \\
\hline 7 & 14 & 1.11 & 0.82 & 0.37 \\
\hline 7 & 15 & 0.02 & 0.24 & 0.41 \\
\hline 8 & 14 & $<0.01$ & 0.02 & 0.05 \\
\hline 8 & 15 & 0.02 & 0.56 & 0.28 \\
\hline Primary & & 32.61 & 0.05 & $<0.01$ \\
\hline Total & & 43.16 & 17.84 & 28.35 \\
\hline
\end{tabular}




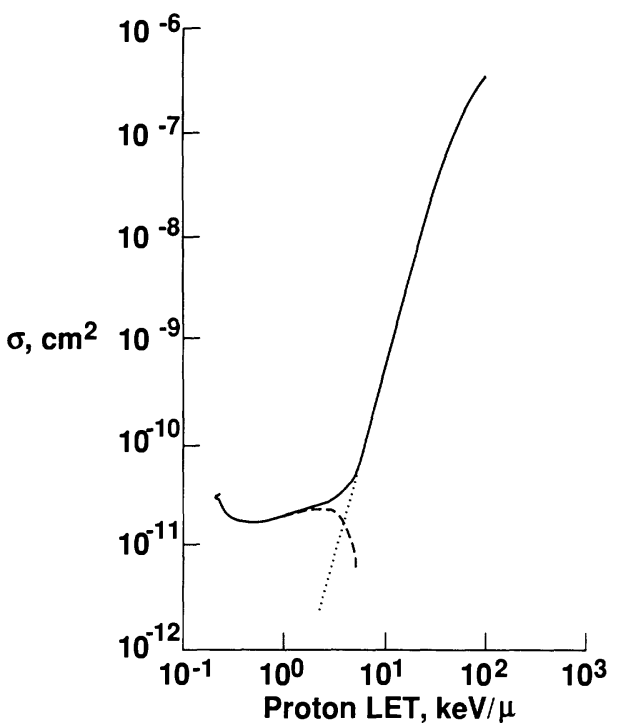

FIG. 3. Calculated values of the proton action cross section for survival of Chinese hamster cells as a function of the proton LET. Lines same as in Fig. 2.

the proton fluence is found as $F_{\mathrm{p}}=6.24 D / \mathrm{LET}$, with $F_{\mathrm{p}}$ in particles $/ \mu \mathrm{m}^{2}$, in Figs. 6 and 8 . The rise in RBE at low dose or fluence, where single proton tracks dominate, is directly attributed to ion kill from both primary protons at $10 \mathrm{MeV}$ and nuclear fragments at the higher energies. Not shown are RBE calculations neglecting the target fragments that are nearly identical to the results for $10-\mathrm{MeV}$ protons shown in Fig. 8, and are almost identical to unity for the 100- and $1000-\mathrm{MeV}$ protons. The relationship of RBE to dose can be seen from Eq. (10), where for $m=3$, using the data of Skarsgard et al. (24), Katz et al. (14) and Katz and Cucinotta (17) have found an RBE dependence on $D^{-2 / 3}$. This effect is supported experimentally as discussed below. An increasing RBE with decreasing dose has also been observed for low-energy neutron irradiations (26). Both ef-

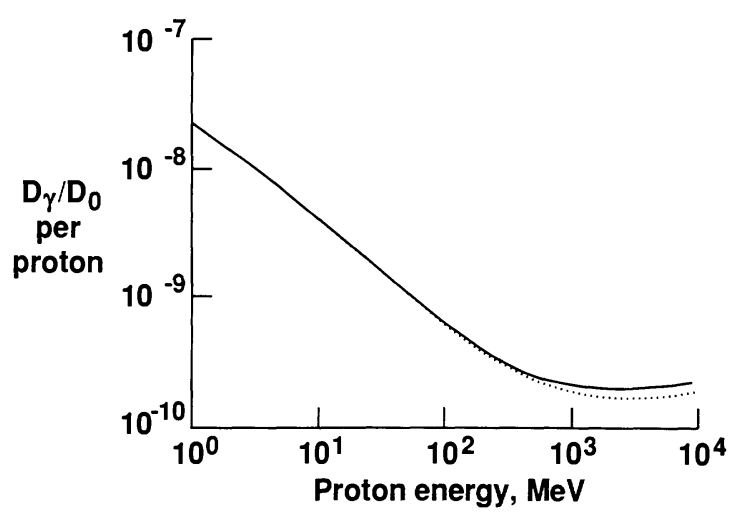

FIG. 4. Calculated values of the proton gamma-kill dose $\left(D_{\gamma} / D_{0}\right)$ for survival of Chinese hamster cells as a function of the proton energy. The dotted line is the primary proton contribution and the solid line includes contributions from fragmentation.

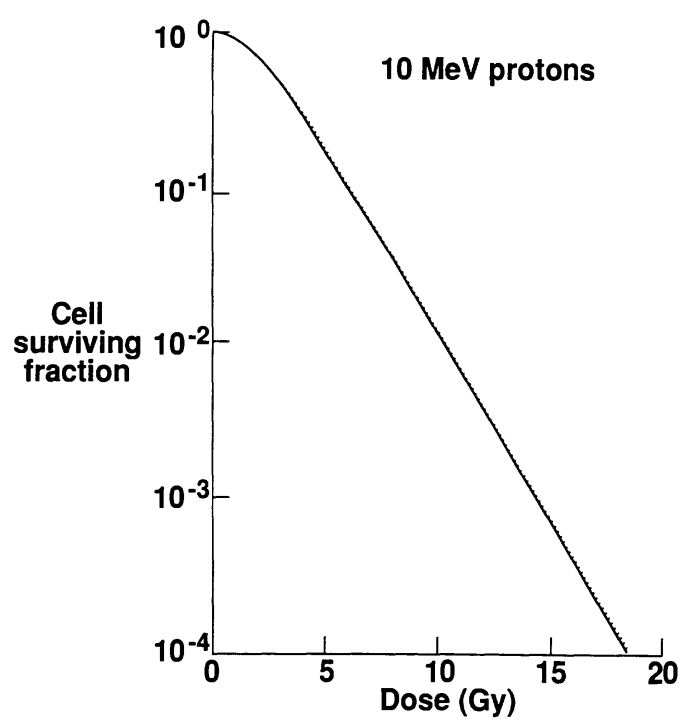

FIG. 5. Calculated cell surviving fraction of Chinese hamster cells for $10-\mathrm{MeV}$ protons. The dashed line is the primary proton and the solid line includes the effects of nuclear fragments.

fects can be attributed to contributions of the production of secondary ions to the ion-kill mode.

\section{EFFECTIVENESS OF 160-MeV PLATEAU- REGION PROTONS}

Cell survival experiments have been performed at the Harvard Cyclotron to determine the biological effectiveness of the protons. In Ref. (10), V79 Chinese hamster cells cultured in vitro were irradiated in the plateau region of the Bragg curve and in a spread-out Bragg peak. Here we compared the survival measurements and RBE determinations for attached cells in the plateau region of the $160-\mathrm{MeV}$ proton Bragg curve using the track model and the high-energy nucleon transport code BRYNTRN (16).

The nucleon transport code BRYNTRN solves the coupled proton-neutron transport problem for high energies in the straight-ahead approximation with multiple-scattering and straggling effects ignored. Target fragments with $A>1$ are transported using the production collision density as given in Eq. (11). The Boltzmann equations for proton and neutron transport are (16)

$$
\begin{aligned}
& {\left[\frac{\partial}{\partial x}-\frac{\partial}{\partial E} S_{\mathrm{p}}(E)+\Sigma_{\mathrm{p}}(E)\right] \phi_{\mathrm{p}}(x, E) } \\
&=\sum_{j} \int_{E}^{\infty} f_{\mathrm{p} j}\left(E, E^{\prime}\right) \phi_{j}\left(x, E^{\prime}\right) d E^{\prime}
\end{aligned}
$$

and

$$
\left[\frac{\partial}{\partial x}+\Sigma_{\mathrm{n}}(E)\right] \phi_{\mathrm{n}}(x, E)=\sum_{j} \int_{E}^{\infty} f_{\mathrm{nj}}\left(E, E^{\prime}\right) \phi_{j}\left(x, E^{\prime}\right) d E^{\prime}
$$

where $\phi_{j}$ is the differential flux of type $j$ particles at $x$ with energy $E ; S_{\mathrm{p}}(E)$ is the proton stopping power; $\Sigma_{\mathrm{p}}(E)$ and 


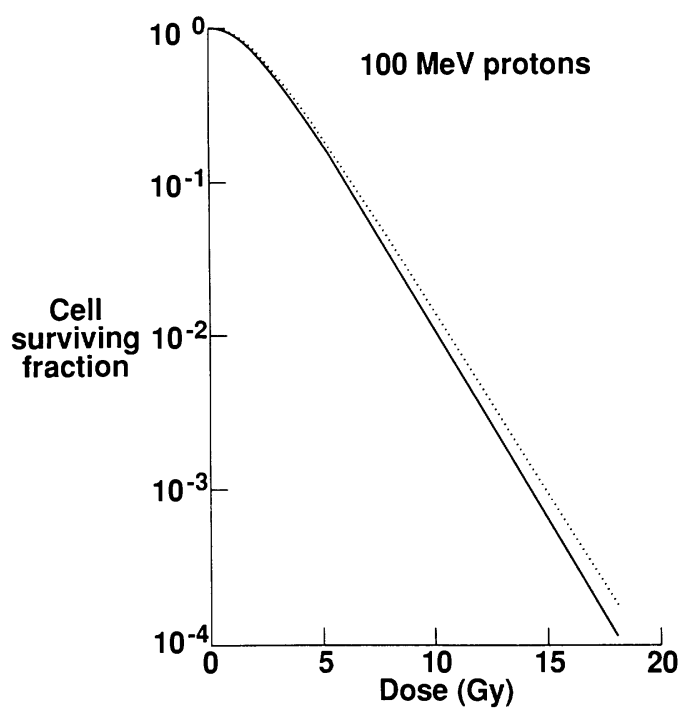

FIG. 6. Calculated cell surviving fraction of Chinese hamster cells for $100-\mathrm{MeV}$ protons. The dashed line is the primary proton and the solid line includes the effects of nuclear fragments.

$\Sigma_{\mathrm{n}}(E)$ are proton and neutron total cross sections, respectively; and $f_{i j}\left(E, E^{\prime}\right)$ represents the differential cross sections for elastic and inelastic processes. As described in Refs. (16, 17), the Boltzmann equations (13) and (14) are solved using a characteristic transformation to reduce the problem to a set of coupled integral equations with boundary conditions at $x=0$, which are then solved numerically. More details on the method of solution and the data base for nuclear scattering are found in Ref. (16). The Bragg curve obtained from BRYNTRN for $160-\mathrm{MeV}$ protons in water is shown in Fig. 9 compared to the measurements of Verhey et al.

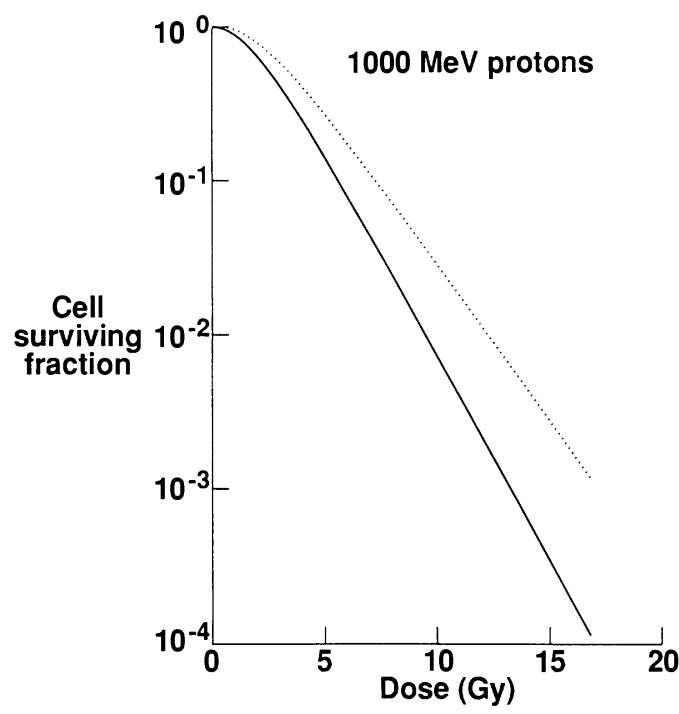

FIG. 7. Calculated cell surviving fraction of Chinese hamster cells for $1000-\mathrm{MeV}$ protons. The dashed line is the primary proton and the solid line includes the effects of nuclear fragments.

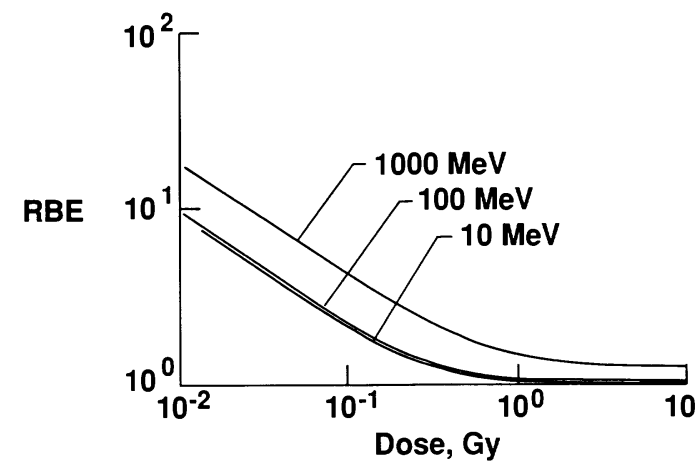

FIG. 8. Calculated proton RBEs for survival of Chinese hamster cells as a function of the absorbed dose. In increasing order: 10-, 100-, 1000$\mathrm{MeV}$ protons.

(28) (solid line). In Fig. 9, the squares represent the primary dose and the circles the total dose with secondary production included. Calculations are normalized to the peak of the experimental Bragg curve. Straggling and multiple-scattering effects, which are not included here, both contribute significantly at the peak of the Bragg curve. We consider the plateau region where the assumptions for high-energy protons are approximately true.

At $160 \mathrm{MeV}$, nuclear recoils from elastic scattering provide a sizable correction to the production of secondary ions represented by target fragmentation. Elastic scattering is represented by the Born term to the optical model renormalized to the total scattering cross section in the BRYNTRN code. This representation of elastic scattering is fairly accurate for integral quantities above $100 \mathrm{MeV}$, but breaks down at lower energies because of multiple scattering, nuclear-medium corrections, and coulomb effects. The correction of the action cross section for protons, taking into consideration the elastic scattering, is shown in Table II for several energies and is included in the following comparisons.

Results for the surviving fraction of V79 Chinese hamster

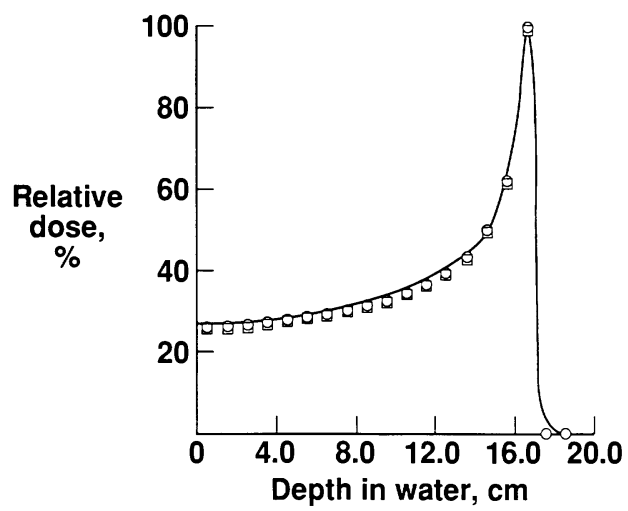

FIG. 9. Depth-dose curve for unmodulated $160-\mathrm{MeV}$ proton beam in water. The measurements of Verhey et al. (28) (solid line) are compared to calculations with nuclear secondaries $(O)$ and without secondaries $(\square)$. 
TABLE II

Secondary Ion Production Contribution to Proton Action Cross Section for V79 Chinese Hamster Cells

\begin{tabular}{ccc}
\hline & \multicolumn{2}{c}{$\sigma\left(10^{-11} \mathrm{~cm}^{2}\right)$} \\
\cline { 2 - 3 }$E(\mathrm{MeV})$ & Elastic recoils & Fragmentation \\
\hline 100 & 1.13 & 1.08 \\
150 & 0.82 & 1.02 \\
1000 & 0.58 & 2.09 \\
\hline
\end{tabular}

cells irradiated in suspension in the plateau region of a 160 $\mathrm{MeV}$ proton beam are shown in Fig. 10. The dashed line is the fit to the $\gamma$-ray survival curve, the dotted line (barely distinguishable from the response for $\gamma$ rays) is the contribution from primaries only, and the solid line includes the calculated effects of nuclear reactions. The characteristic $\gamma$-ray dose, $D_{0}$, is taken as $2.9 \mathrm{~Gy}$ to reproduce the experimental curve for $\gamma$ rays with the other response parameters given above. The survival curves for Chinese hamster cells irradiated with ${ }^{60} \mathrm{Co} \gamma$ rays and plateau-region $160-\mathrm{MeV}$ protons are similar, indicating that, when the effects of the nuclear force are disregarded, the behavior of high-energy protons is like that of $\gamma$ rays. Agreement with the data is fair, indicating that the modeling of nuclear fragmentation made here is somewhat lacking. In Fig. 11, we compare our results for the proton RBE (solid line) against the values obtained using the analysis methods of Kellerer and Brenot (vertical bars) (29) in Ref. (10). The "bare" proton RBE has a value of 1 (not shown), except at the lowest doses where a small contribution from ion kill gives a slight increase. The calculations of the proton RBE presented in Fig. 11 show

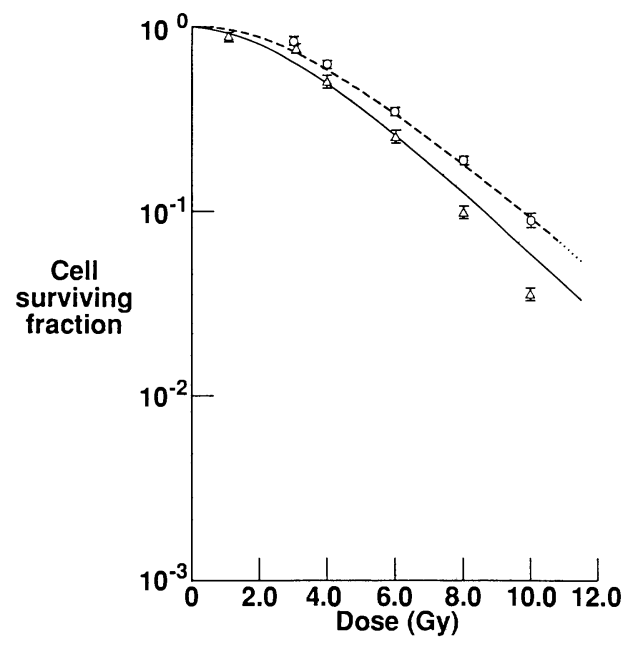

FIG. 10. Survival data for Chinese hamster cells irradiated by ${ }^{60} \mathrm{Co} \gamma$ rays $(O)$ and plateau-region $160-\mathrm{MeV}$ protons $(\Delta)$ from Hall et al. $(10)$ are compared to calculations. The dashed line is the fit to the experimental $\gamma$-ray survival curve, the dotted line is the result for primary protons only, and the solid line includes the effects of nuclear reactions.

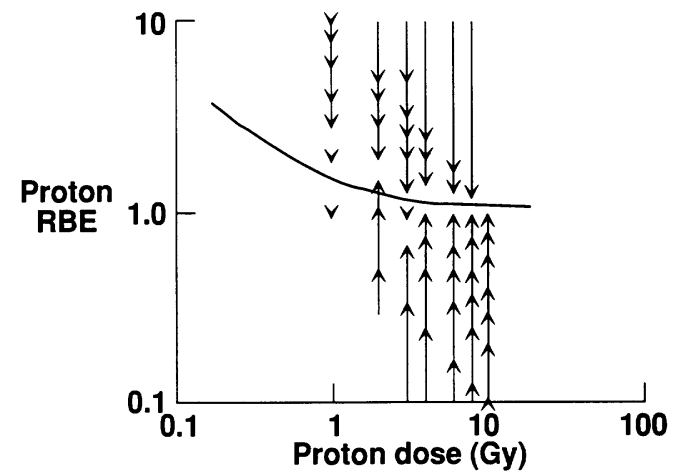

FIG. 11. Proton RBE as a function of proton dose for Chinese hamster cell survival in plateau region of the $160-\mathrm{MeV}$ proton Bragg curve. Experimental determination from Hall et al. (10). The vertical bars denote RBE values that are excluded at $95 \%$ confidence level, while the detached arrows indicate values that are unlikely at a lower level of confidence. The most likely RBE values are in the space between the vertical bars. The solid line is from the calculations where the effects of nuclear reactions are included.

good agreement with the values obtained experimentally, with the increasing RBE at low doses shifted to lower values than those obtained by experiment. A second method of analysis, which assumes that the dose-effect curve is convex but is otherwise independent of shape, was used to derive RBE values (10); the values are shown in Fig. 12 in comparison to the present predictions. The RBE rise at low dose as $D^{-2 / 3}$ predicted from Eq. (10) is clearly seen in the calculations (solid line) and the experimental analyses (dashed line). Also shown in Fig. 12 by the dotted line are our calculations neglecting nuclear reactions.

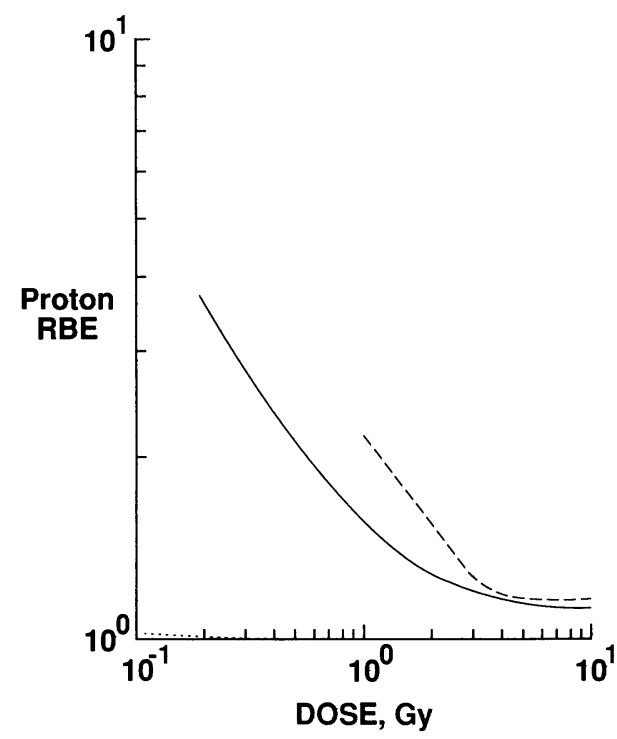

FIG. 12. Proton RBE as a function of proton dose for Chinese hamster cell survival in plateau region of the $160-\mathrm{MeV}$ proton Bragg curve. The dashed line is from experimental analysis in Ref. (10) as discussed in text. The solid line is calculations which include nuclear reaction effects, and the dotted line without nuclear reactions. 


\section{CONCLUSIONS}

The biological effectiveness of high-energy protons, when cellular damage proceeds through a multitarget response $(m>1)$, has been identified to be related intimately to the production of nuclear fragments in tissue along the track of the protons. Single tracks of high-energy protons produce an insignificant contribution to the action cross section (see Fig. 2); it is only through secondary nuclear reactions that damage occurs. The increase in RBE at low doses found in experiments with fast heavy ions is also found for protons because of the consequences of nuclear scattering. A doseor fluence-dependent proton RBE has important consequences for radiation exposures in space from high-energy protons. The magnitude of the RBE is related to the curvilinear response curve of the reference radiation. The curvilinearity, in contrast to the linear response for high-LET radiation, reflects the repair of low-LET radiation-induced damage. Our calculations suggest that an understanding of the effects of nuclear interactions in tissue is essential for risk assessment for high-energy protons, especially at low levels of exposure.

\section{ACKNOWLEDGMENT}

This work was supported in part by the Armed Forces Radiobiology Research Institute under N91 R0001 and by the United States Department of Energy.

RECEIVED: December 4, 1990; ACCEPTED: February 28, 1991

\section{REFERENCES}

1. A. Bouville and W. M. Lowder, Human population exposure to cosmic radiation. Radiat. Prot. Dosim. 24, 293-299 (1988).

2. R. J. M. FRY and D. S. NACHTWEY, Radiation protection guidelines for space missions. Health Phys. 55, 159-164 (1988).

3. NCRP, Guidance on Radiation Received in Space Activities, Report No. 98. National Council on Radiation Protection and Measurements, Bethesda, MD, 1989.

4. R. J. M. Fry, P. Powers-Risius, E. L. Alpen, and E. J. AINSWORTH, High-LET radiation carcinogenesis. Radiat. Res. 104, S188-S195 (1985).

5. T. C. YANG, L. M. MeI, W. MeI, and C. A. Tobias, Neoplastic cell transformation by heavy charged particles. Radiat. Res. 104, S177S187 (1985).

6. T. C. YANG and C. A. ToBIAS, Neoplastic cell transformation by energetic heavy ions and its modification with chemical agents. $A d v$. Space Res. 4, 207-218 (1984).

7. J. B. Robertson, J. R. Williams, R. A. SChmidt, J. B. Little, D. F. FLYNN, and H. D. SUIT, Radiobiological studies of high-energy modulated proton beams utilizing cultured mammalian cells. Cancer 35, 1664-1677 (1975).

8. M. Urano, M. Goitein, L. Verhey, O. Mendiondo, H. D. Suit, and A. KOEHLER, Relative biological effectiveness of a high energy modulated proton beam using a spontaneous murine tumor in vivo. Int. J. Radiat. Oncol. Biol. Phys. 6, 1187-1193 (1980).

9. J. TEPPER, L. Verhey, M. GoITEIN, and H. D. SUIT, In vivo determi- nations of RBE in high energy modulated proton beams using normal tissue reactions and fractionated dose schedules. Int. J. Radiat. Oncol. Biol. Phys. 2, 1115-1122 (1977).

10. E. J. Hall, A. M. Kellerer, H. H. Rossi, and Y. P. Lam, The relative biological effectiveness of $160 \mathrm{MeV}$ protons. II. Int. J. Radiat. Oncol. Biol. Phys. 4, 1009-1013 (1978).

11. G. Barendsen, G. W. Walter, H. M. D. Fowler, and D. D. BewLEY, Effects of different ionizing radiations on human cells in tissue culture. Radiat. Res. 18, 106-119 (1963).

12. R. Katz, R. Ackerson, M. Homayoonfar, and S. C. Sharma, Inactivation of cells by heavy ion bombardment. Radiat. Res. 47, 402-425 (1971).

13. R. Katz, S. C. Sharma, and M. Homayoonfar, The structure of particle tracts. In Topics in Radiation Dosimetry (F. H. Attix, Ed.). Academic Press, New York, 1972.

14. R. Katz, D. E. DunN, and G. L. Sinclair, Thindown in radiobiology. Radiat. Prot. Dosim. 13, 281-284 (1985).

15. J. W. WILSON, L. W. TOWNSEND, and F. KHAN, Evaluation of highly ionizing components in high-energy nucleon radiation fields. Health Phys. 57, 717-724 (1989).

16. J. W. Wilson, L. W. TOWNSEND, S. Y. Chun, W. W. BuCK, F. KHAN, and F. A. CUCINOTTA, BRYNTRN: A Baryon Transport Computer Code. NASA TM 4037 (1988); also NASA TP 2887 (1989).

17. R. KATZ and F. A. CUCinotTA, RBE vs. dose for low doses of high LET radiations. Health Phys., 60, 717-718 (1991).

18. J. W. WILSON, Analysis of the Theory of High-Energy Transport, Report TN D-8381 1. NASA, Washington, DC, 1977.

19. H. W. BERTINI, MECC7: A Monte Carlo Intranuclear Cascade and Evaporation Code. Radiation Shielding Information Center, Oak Ridge National Laboratory, Oak Ridge, TN, 1970.

20. R. Silberberg, C. H. Tsao, and M. M. Shapiro, Semiempirical cross sections. In Spallation Nuclear Reactions and Their Applications (B. S. P. Shen and M. Merker, Eds.), pp. 49-81. Dardrecht, Boston, 1976.

21. D. E. Greiner, P. J. Linstrom, H. A. Heckman, B. Cork, and F. S. BIESER, Momentum distributions of isotopes produced by relativistic 12-C and 16-O projectiles. Phys. Rev. Lett. 35, 152-155 (1975).

22. J. W. WILSON, L. W. TownSEND, and F. F. BADAVIA, Galactic HZE propagation through the earth's atmosphere. Radiat. Res. 109, 173183 (1987).

23. J. F. ZIEGLER, Stopping Cross-Sections for Energetic Ions in All Elements. Pergamon, New York, 1980.

24. L. D. Skarsgard, B. A. Kihlman, L. Parker, C. M. Pujara, and S. RICHARDSON, Survival chromosome abnormalities, and recovery in heavy-ion and X-irradiated mammalian cells. Radiat. Res. Suppl., 7, 208-221 (1967).

25. F. A. Cucinotta, F. Hajnal, and J. W. Wilson, Energy deposition at the bone-tissue interface from target fragments produced by high energy nucleons. Health Phys. 59, 819-825 (1990).

26. ICRU, The Quality Factor in Radiation Protection, Report 40. International Commission on Radiation Units and Measurements, Bethesda, MD, 1986.

27. J. W. WILSON and S. L. LAMkIN, Perturbation theory for chargedparticle transport in one dimension. Nucl. Sci. Eng. 57, 292-299 (1975).

28. L. J. Verhey, A. M. Koehler, J. C. MCDonald, M. Goitein, I-C. MA, R. J. SCHNEIDER, and M. WAGNER, The determination of absorbed dose in a proton beam for purposes of changed-particle radiation therapy. Radiat. Res. 79, 34-54 (1979).

29. A. M. Kellerer and J. BRENOT, Nonparametric determination of modifying factors in radiation action. Radiat. Res. 56, 28-39 (1973). 